Fonum .

Sociológico

\section{Forum Sociológico}

Série II

32 | 2018

Fraturas sociais e educativas: Desafios para a sociologia da educação

\title{
Las experiencias escolares como marco interpretativo de las desigualdades socio- educativas en la educación secundaria : Una aproximación teórica
}

Experiências escolares como quadro interpretativo para desigualdades sócioeducativas no ensino secundário : Uma abordagem teórica School experiences as an interpretative framework of socio-educational inequalities in secondary education: A theoretical approach

Francisco Santana-Armas, María del Mar Noda-Rodríguez y Carmen Nieves Pérez-Sánchez

\section{(2) OpenEdition}

\section{Edición electrónica}

URL: https://journals.openedition.org/sociologico/1960

DOI: 10.4000/sociologico.1960

ISSN: 2182-7427

\section{Editor}

CICS.NOVA - Centro Interdisciplinar de Ciências Sociais da Universidade Nova de Lisboa

Edición impresa

Paginación: 29-37

ISSN: 0872-8380

Referencia electrónica

Francisco Santana-Armas, María del Mar Noda-Rodríguez y Carmen Nieves Pérez-Sánchez, «Las experiencias escolares como marco interpretativo de las desigualdades socio-educativas en la educación secundaria : Una aproximación teórica», Forum Sociológico [En línea], 32 | 2018, Puesto en línea el 16 julio 2018, consultado el 29 marzo 2022. URL: http://journals.openedition.org/sociologico/ 1960 ; DOI: https://doi.org/10.4000/sociologico.1960 


\title{
LAS EXPERIENCIAS ESCOLARES COMO MARCO INTERPRETATIVO DE LAS DESIGUALDADES SOCIO-EDUCATIVAS EN LA EDUCACIÓN SECUNDARIA: UNA APROXIMACIÓN TEÓRICA ${ }^{1}$ \\ EXPERIÊNCIAS ESCOLARES COMO QUADRO INTERPRETATIVO PARA DESIGUALDADES SÓCIO-EDUCATIVAS NO ENSINO SECUNDÁRIO: UMA ABORDAGEM TEÓRICA
}

SCHOOL EXPERIENCES AS AN INTERPRETATIVE FRAMEWORK

OF SOCIO-EDUCATIONAL INEQUALITIES IN SECONDARY EDUCATION:

A THEORETICAL APPROACH

Francisco Santana-Armas

- Universidad de La Laguna, Departamento de Sociología y Antropología, Tenerife, Islas Canarias, España

María del Mar Noda-Rodríguez

- Universidad de La Laguna, Departamento de Sociología y Antropología, Tenerife, Islas Canarias, España

Carmen Nieves Pérez-Sánchez

Universidad de La Laguna, Departamento de Sociología y Antropología, Tenerife, Islas Canarias, España

\begin{abstract}
Resumen
Si hay una temática que preocupa especialmente a la Sociología de la Educación, esta es la relación entre la desigualdad social y los desiguales resultados educativos. Indagamos, inicialmente, sobre las visiones que analizan los déficits culturales del alumnado; la naturaleza del sistema educativo que corresponde con una cultura de clase media y la asociación entre origen social y rendimiento educativo. Posteriormente, consideramos que, para explicar los mecanismos de legitimación o cambio social, y el papel que juega en ello la educación - y de manera especial en la educación secundaria -, es preciso no sólo tener en cuenta las desigualdades socio-económicas del alumnado sino también sus experiencias escolares, construidas en su trayectoria y espacio escolar. La configuración analítica desde estas premisas nos ayuda a contextualizar las experiencias escolares como un todo complejo, a veces contradictorio, pero nos permite estudiar mejor los desiguales resultados educativos. En este sentido, constatamos que la "experiencia escolar" podría ser un concepto-puente que aglutina las interacciones entre las culturas sociales representadas por la diversidad socio-cultural del alumnado y la cultura escolar.
\end{abstract}

Palabras claves: experiencia escolar, desigualdad educativa, cultura escolar, educación secundaria

\section{Resumo}

Se existe um tema que é particularmente preocupante para a sociologia da educação, esta é a relação entre desigualdade social e resultados educacionais desiguais. Perguntamos, inicialmente, sobre as visões que analisam os déficits culturais dos alunos; a natureza do sistema educacional que corresponde a uma cultura de classe média e a associação entre origem social e desempenho educacional. Posteriormente, consideramos que, para explicar os mecanismos de legitimação ou mudança social, e o papel que a educação desempenha neste - e especialmente no ensino secundário -, é necessário não só levar em conta as desigualdades socioeconômicas dos alunos, mas também suas experiências escolares, construídas sobre sua trajetória e espaço escolar. A configuração analítica dessas premissas nos ajuda a contextualizar as experiências escolares como um todo complexo, às vezes contraditório, mas nos permite estudar melhor os resultados educa- 
cionais desiguais. Nesse sentido, achamos que a "experiência escolar" pode ser um conceito de ponte que reúne as interações entre culturas sociais representadas pela diversidade sociocultural do corpo estudantil e a cultura escolar.

Palavras-chave: experiência escolar, desigualdade educacional, cultura escolar, ensino secundário

\begin{abstract}
If there is a subject for which Sociology of Education is concerned about, this is the relationship between social inequality and unequal educational outcomes. We investigate, initially, the visions that analyze the cultural deficits of students; the nature of the educational system that corresponds to a middle-class culture and the association between social origin and educational performance. Subsequently, we consider that to explain the mechanisms of legitimation or social change, and the role that education plays in it - and especially in secondary education -, is necessary not only to take into account the socio-economic inequalities of students but also their school experiences, built in its trajectory and school space. The analytical configuration from these premises helps us to contextualize the school experiences as a whole complex, sometimes contradictory, but allows us to study better the unequal educational results. In this sense, we verify that the "school experience" could be a bridge-concept that brings together the interactions between the social cultures represented by the socio-cultural diversity of the students and the school culture.
\end{abstract}

Keywords: school experience, educational inequality, school culture, secondary education

\section{Antecedentes}

Conocer las razones y causas del éxito, fracaso y abandono escolar es un objetivo que ha estado presente en el origen y desarrollo de la Sociología de la Educación. Además, se ha convertido en uno de los temas centrales en las interpretaciones de los resultados de los diagnósticos que se han desarrollado en el caso español y en los países de nuestro entorno (Organización para la Cooperación y Desarrollo Económico) desde finales del siglo XX, además de factor destacado en los argumentos que justifican los cambios en las políticas educativas. Más si cabe cuando el efecto masificador que tuvieron determinadas políticas, vinculadas a potenciar el ideal de la igualdad de oportunidades, a posteriori, ha supuesto el desarrollo de fuertes procesos de diversificación y ramificación de los sistemas educativos, fundamentalmente en la educación secundaria. Estos procesos de diversificación y selección según el rendimiento del alumnado han provocado igualmente un claro aumento de la competencia escolar dentro de los centros y llegado el caso entre los centros. Así los procesos de competencia escolar que estaban condicionados por la selección social antes de la incorporación a los sistemas educativos, se desplazan al interior del sistema que tiene como efecto que las variables vinculadas a los condicionantes sociales y culturales sigan siendo de interés fundamental (Dubet y Martuccelli, 1998).

Los efectos de la selección escolar y sus derivas, analizados desde el interior del sistema, permiten abrir nuevas perspectivas que incorporan al debate sobre las desigualdades la problemática de la justicia escolar y aquellos aspectos relativos a la introducción de una visión eufemística de las desigualdades al calificarlas de justas centrando el análisis en el grado de igualdad y no en el de justicia (Derouet, 2002; 2005; Derouet y Normand, 2011; Resende, 2010). El papel del profesorado, en este marco de análisis, es sumamente importante como agente político. Lo es, además, porque el profesorado es uno de los agentes que parece haber asumido las premisas ideológicas sobre las funciones reproductoras, en sus distintas versiones, (Cabrera, Báez, Cabrera, Noda, Pérez, Santana y Zamora, 2011; Tarabini, 2015) del sistema educativo. En este sentido, la evolución de las políticas educativas, no han ayudado a poner en primera línea un debate profundo sobre las funciones del sistema educativo. Todos, políticos y profesionales (no sólo el profesorado sino también parte del análisis sociológico), parecen asumir la creencia de la relación entre educación, crecimiento económico y desarrollo social, aceptando nuevas racionalidades que cubren ideológicamente la necesidad de que la escuela actúe de acuerdo a la evolución de la organización y de las condiciones de trabajo del capitalismo, contribuyendo a la preparación del alumnado para una vida ordenada y de convivencia adecuada.

En el contexto actual, la meta ideológica en educación tiene que ver con una visión cortoplacista del éxito educativo y de lograr, bajo el paraguas de la calidad y del esfuerzo individual, mejores resultados educativos. No es que esto sea negativo en sí mismo, lo que aquí nos interesa es cómo esa idea madre de la política educativa actual bebe de un discurso con una fuerte lógica y racionalidad economicista, 
en donde las ideas de eficacia y eficiencia (Angus, 1993; Ball, 1997; Brown y Tannock, 2009) son fuente discursiva para legitimar el discurso político actual y para el caso que nos ocupa, olvidar o minusvalorar las prácticas educativas en las diversas y desiguales realidades escolares. La diversidad reconocida en este tipo de racionalidades se visibiliza a partir de las diferencias individuales y no así a partir de las desigualdades sociales del alumnado.

Aun así, todos los estudios - tanto académicos como institucionales - indican la permanente vinculación entre el origen social y el rendimiento académico, y ello a pesar de la implementación de medidas o modelos educativos supuestamente diseñados para desarrollar mecanismos de inclusión social, como se mencionaba anteriormente. Pero no todas las variables asociadas a la desigualdad social han tenido el mismo recorrido. De las reformas emprendidas para paliar directa o indirectamente las exclusiones o segregaciones de distintos grupos sociales, ha sido la desigualdad de género la que ha conseguido cuotas más altas de éxito y transformación.

En cualquier caso, y siendo conscientes de que cuando hablamos de desigualdad social estamos refiriéndonos a una intersección de realidades sociales, económicas y culturales, encontramos sobrada bibliografía en Sociología de la Educación para abordar gran parte de la problemática que nos preocupa y ocupa: la articulación entre desigualdades sociales y educativas. Sintéticamente destacamos tres grandes perspectivas analíticas:

1) Una perspectiva en auge y que tiene que ver con la proliferación de estudios cuantitativos sobre los resultados escolares del alumnado y la evidencia empírica de que son las variables relacionadas con las condiciones de partida (fundamentalmente nivel de estudios de los progenitores) las que influyen en mayor medida en las trayectorias escolares. No existen intentos serios por traducir en términos de teorización estos resultados que permita insertarlos en una construcción de la complejidad social y, en consecuencia, en debates sobre modelos de sociedad y de educación (Cabrera, Cabrera, Pérez y Zamora, 2011; Carmona Rodríguez, 2017; Dubet, 2000).

2) El menor éxito escolar del alumnado de grupos subalternos se explica fundamentalmente por los déficits culturales o motivacionales del alumnado y por las formas poco adecuadas de socialización familiar. En este sentido, una parte importante de las investigaciones en torno a las formas de socialización familiar son etnocéntricas (Martín Criado, Gómez Bueno, Fernández Palomares y Rodríguez Monge, 2000).
En general, domina una conceptualización deficitaria sobre la socialización familiar de la clase obrera, bien en términos de aspiraciones de logro, de códigos lingüísticos o de prácticas educativas. Además, a la hora de explicar el fracaso escolar, las limitaciones del alumnado y sus familias son un registro presente también en la lógica institucional, encontrando en las voces del profesorado (Pérez Sánchez y Noda Rodríguez, 2012) una perspectiva etnocéntrica y legitimista. En una investigación dirigida por Cabrera, Báez, Cabrera, Noda, Pérez, Santana y Zamora (2011) sobre las mentalidades y el pensamiento del profesorado no universitario en Canarias $^{2}$, descubrimos que de los factores que más influyen en los malos resultados del alumnado canario se encuentran, según sus percepciones, la falta de responsabilidad y disposición para el esfuerzo por parte del alumnado y la despreocupación de los padres por la educación de los hijos. Y ello a pesar de que la clase obrera ha hecho unos esfuerzos importantes en los procesos de implicación en el mundo escolar (Martín y Bruquetas, 2014). A esto se une el hecho de que normalmente el profesorado tiene interiorizada una valoración negativa sobre las culturas de los jóvenes subalternos y sus circunstancias y que parece solamente pueden ser superadas y compensadas por una clara adaptación del alumnado y sus familias a la acción educativa, entendida desde los parámetros de la institución escolar (Giró y Andrés, 2016; Grignon, 1991; Martín Criado, 2004).

3) La desigualdad de resultados escolares en relación al origen social se explica por la propia naturaleza y estructura del sistema educativo. El planteamiento general viene a ser el siguiente: la educación no puede ser igualitaria porque nuestra sociedad está conformada a partir de la desigualdad social, económica y cultural. Con lo que la escuela actual, a pesar de que quiera comportarse como una institución de masas, no puede evitar desarrollar una cultura que se corresponde con la cultura de clase media (Cabrera, 1991; Collet Sabé, 2013). $Y$ es que la escuela no fue históricamente construida para trabajadores, mujeres ni minorías étnicas. En su conformación, se convierte en hegemónica la cultura, actitudes, valores, comportamientos de los grupos dominantes (Fernández Enguita, 1997, p. 112). Con mayor precisión, para Bourdieu y Passeron (1977) la acción pedagógica tiende a reproducir la estructura de 
clases, imponiendo (Violencia Simbólica) la cultura de las clases dominantes como legítima. Las relaciones entre la escuela y las familias obreras son claramente asimétricas, donde los alumnos y alumnas tienen, básicamente, dos opciones: se alejan de la cultura escolar (fracaso escolar, abandono escolar; trayectorias académicas cortas; itinerarios prácticos); o se integran en la cultura académica, alejándose de la cultura propia (el premio es el éxito escolar y trayectorias académicas largas). También para Bernstein (1988) los conocimientos, los lenguajes y las pedagogías desarrolladas y valoradas en la escuela son elementos asumidos como propios por parte de las clases media.

En otra línea, se han desarrollado otros trabajos, desde una perspectiva micropolítica, que analizan empíricamente la importancia de los centros escolares en la gestión de las oportunidades educativas (Merino, García, Torrent y Valls, 2017; Tarabini, Curran, Montes y Parcerisa, 2015).

Desde nuestro punto de vista, la asociación entre el origen social y el rendimiento académico puede explicarse en términos de afinidades $\mathrm{y} / \mathrm{o}$ distancias culturales. Desde esta perspectiva, el núcleo interpretativo fuerte son las relaciones entre las culturas sociales representadas por el origen sociocultural del alumnado y la cultura escolar. En este sentido resulta necesario analizar tanto las concepciones del alumnado y sus familias como las formas de la cultura escolar, las justificaciones del profesorado sobre las desigualdades escolares y las trayectorias futuras del alumnado.

\section{Experiencias escolares e identidades juveniles}

Utilizamos la noción de "experiencia escolar" como concepto-puente que aglutina las interacciones entre las culturas sociales representadas por la diversidad sociocultural del alumnado y la cultura escolar. Son varios los autores que incorporamos a este enfoque.

En una de sus primeras aproximaciones, Dubet $^{3}$ (2010, p. 96) nos habla de una sociología de la experiencia social, en donde la misma es una "combinación de lógicas de acción". El actor lleva a cabo esas lógicas diversas a través de sus conductas y percepciones de una realidad determinada, constituyendo su propia subjetividad y reflexividad. Entendemos también con Dubet y Martuccelli (1998) que la experiencia escolar, por tanto, no es un objeto que se observe y mida desde fuera porque se trata de un trabajo del actor que define una situación, elabora jerarquías de selección y construye imágenes de sí mismo. Interpretan que la socialización "es un proceso paradójico, por una parte, es un proceso de inculcación; por otra, sólo se realiza en la medida en que los actores se constituyen como sujetos capaces de manejarla" (Dubet y Martuccelli, 1998, p. 15).

Este enfoque nos ayuda a superar, desde un punto de vista analítico, una mirada delimitada y condicionada por las políticas vinculadas al rendimiento académico que se centran, exclusivamente, por metas cuantitativas donde el alumnado era es - un mero número que debería alcanzar unos objetivos relacionados con una visión resultadista del conocimiento escolar. Los análisis académicos, incluso los más críticos, a menudo olvidan las percepciones particulares de ese alumnado, haciendo hincapié en algo sin duda importante pero no suficiente para un análisis global, cual era las potenciales desigualdades educativas que este tipo de políticas acarreaba. Esta visión macro, no relata claramente la visión propia de los sujetos tanto en la implementación de esas políticas como las consecuencias de las mismas, vehiculadas hacia una visión de los alumnos en relación a su inteligencia y sus capacidades, siendo básicamente una cuestión de "conductas", lo que evidencia un desinterés por la contextualización y explicación de las acciones y prácticas de los actores (Dubet, 2000).

Desde otra aproximación, asumimos con Willis $(1988 ; 2008)$ el sentido activo de los sujetos que condicionados pero no determinados por sus condiciones sociales construyen sus propios sistemas de valores, gustos y concepciones vitales.

Tanto para uno como para otros los institutos son lugares de aprendizaje pero también de vida juvenil. Efectivamente para Dubet y Martuccelli (1998) las experiencias escolares "se fabrican" en el seno de las escuelas y son el resultado de las formas en las que las escuelas "han tratado" al alumnado pero también de lo mucho que se les escapa a su control. Y para Willis "debe entenderse que la escolarización no sólo es un instrumento para producir modernidad, es también un sitio para el despliegue de sus formas y fuerzas contradictorias" (Willis, 2008, p. 63).

Al centrar el objeto de estudio en las experiencias escolares le damos importancia a los espacios donde se construyen, lo que nos permite comprender más profundamente los procesos sociales y políticos, desde los propios actores. Dentro de los institutos se dan respuestas claramente identificadas con la cultura escolar pero también muchos otros esquemas de valores o sistemas de estatus (de medición y logro) vinculados a las culturas juveniles o culturas populares. Por ejemplo, dentro de estas últimas: sistema de estatus basados en el deporte; el atractivo sexual; oposición a la autoridad; nuevos patrones de consumo cultural basado en las redes sociales... 
Existen distintos estudios sobre las experiencias escolares y la desigualdad social: en el ámbito español centrados en los estudiantes universitarios (Langa Rosado, 2006) o en el alumnado de clase trabajadora (Pérez Sánchez, 2002); en el caso de Cataluña en relación al alumnado de enseñanza media (Bonal, 2005). Distintos investigadores argentinos han centrado también sus estudios en las experiencias del alumnado de secundaria de distintas clases sociales (Duschatzky, 1999; Kessler, 2002; Núñez y Litichever, 2015; Sambucceti, 2014); en Francia los trabajos ya mencionados de François Dubet $(2000 ; 2010)$ y en algún caso en coautoría con Danilo Martucelli (1998).

Así, sin lugar a dudas los trabajos de François Dubet $(1998 ; 2000 ; 2010)$, nos ayudan a centrar una perspectiva de análisis que podríamos llamar en un primer momento sociología de la experiencia y en un segundo momento sociología de los actores. Se configura una forma de afrontar explicaciones desde los actores en un espacio social concreto. De ahí y siguiendo los planteamientos de Dubet y Martucelli (1998), analizados por Núñez y Litichever (2015, p. 23):

La experiencia escolar es entendida como una construcción personal, social e institucional, ya que comprende las relaciones, significaciones, lógicas de acción y estrategias a través de las cuales estudiantes y profesores se constituyen en su integración a un espacio escolar con diversas lógicas de acción institucional.

Se establece, por tanto, un espacio de análisis vinculado a presenciar cómo los sujetos construyen de manera subjetiva una suerte de identidad ante el resto de los sujetos y en contraste con el contexto social y escolar cercano. Este planteamiento se aleja en parte de lo macro, en un primero momento, para atender los espacios micros de construcción social propia de significados.

En definitiva, analizar el papel que juega el sistema educativo en los mecanismos de reproducción o modificación social, requiere no sólo tener en cuenta las desigualdades socio-económicas del alumnado sino también sus experiencias escolares, construidas por sus resultados académicos, trayectorias, expectativas escolares y relaciones con sus iguales, familias y profesorado.

Para estudiar las desigualdades socio-económicas debemos tener en cuenta las transformaciones socio-económicas-culturales que han acaecido en las últimas décadas, que han profundizado las desigualdades. Se han tambaleado los mecanismos y estrategias de reproducción social por parte de las familias. Actualmente, incluso, algunos sectores de la clase media se sienten más vulnerables y menos seguros de la reproducción a través de la escuela (Boltanski y Chiapello, 2002, pp. 25-26), aunque son los sectores populares que han tenido históricamente mayores dificultades de rentabilización de los estudios los que sufren más agudamente los procesos de exclusión social (tanto laboral como de oportunidades escolares).

Vivimos en sociedades complejas y con sistemas educativos por ende complejos, en donde es muy difícil establecer el peso exacto de los elementos que influyen en la legitimación o cambio social. Tradicionalmente se ha considerado el resultado educativo, es decir la calificación que obtiene el alumnado al finalizar un curso o etapa educativa, como el mejor indicador e incluso el único, dependiendo de la perspectiva analítica que utilicemos, que explica dicho proceso de reproducción o cambio. Sin embargo, nosotros consideramos que su relevancia debe ser estudiada en el marco de un ámbito más amplio como es el de la experiencia escolar, ya que forma parte de éste. En tanto que motor y resultado de la confluencia entre las motivaciones, percepciones, expectativas, trayectorias, resultados académicos del alumnado y de las relaciones con sus iguales, familia y profesorado, las experiencias escolares constituyen una vía analítica más que significativa para explorar el proceso educativo y sus efectos.

Nos encontramos ante un alumnado que debe ser analizado como un agente activo que construye su experiencia escolar a través de los distintos ámbitos de socialización a los que está sometido y por su respuesta ante las diferentes situaciones y coacciones a las que se enfrenta, en donde es imposible separar su status de alumno de su status de joven. Dicha construcción no es fácil ni mecánica sino compleja y contradictoria por la intersección que se produce entre la cultura social, juvenil y escolar.

Sin embargo, consideramos que, a pesar de dicha dificultad, es imprescindible tratar de explicar qué procesos están teniendo lugar en el sistema educativo para que éste siga siendo más un agente de legitimación que de transformación social. Así, creemos que es vital estudiar la relación entre el origen social del alumnado y su experiencia escolar.

Todos los estudios realizados desde la mitad del pasado siglo corroboran que existe una vinculación entre el origen social del alumnado y el rendimiento educativo, o más concretamente entre el nivel educativo de los padres y el de sus hijos, y ello a pesar de las distintas reformas educativas emprendidas. Lo que nos ha llevado a considerar que el sistema educativo es un factor que, más que dador del cambio, es un legitimador social.

Por tanto, a pesar de que la escuela no sea el eje central a través del cual tiene lugar el cambio social y por ello no haya podido cumplir las grandes expectativas generadas en torno a ella, o dicho de otra forma, aunque las distintas reformas 
educativas emprendidas con el objetivo de lograr la igualdad de oportunidades sociales a través de la igualdad de oportunidades educativas no hayan tenido los resultados esperados, sin embargo es necesario explicar qué procesos tienen lugar en la institución que se ha convertido en uno de los pilares básicos de las sociedades democráticas. Es importante analizar y conocer tanto las experiencias escolares del alumnado como las profesionales de los docentes, estudiar cómo se construyen, cómo se influyen y condicionan mutuamente, así como las características organizativas de los centros que pueden condicionar estos procesos.

\section{Una experiencia escolar contextualizada}

Partimos, por tanto, de una categoría analítica que necesita también ser contextualizada con otros espacios que conforman una misma realidad. Esta delimitación ayuda a abordar de manera coherente aspectos centrales que pueden condicionar las experiencias escolares del alumnado, de ahí que los siguientes apartados pueden configurar los distintos ámbitos de análisis e influencia.

\section{La importancia del escenario escolar}

Empecemos señalando que el papel socializador del centro educativo no se lleva a cabo de forma lineal y coherente, dado los múltiples factores implicados: ubicación, titularidad pública o privada, tamaño, infraestructura, prestigio/estigmatización, política y proyecto educativo, ratios profesorado-alumnado, configuraciones organizativas, etc. Por ello es necesario contrastar la experiencia escolar con, por ejemplo, la relativa influencia que tiene la titularidad del centro y la naturaleza competitiva o cooperativa de los métodos didácticos en los resultados escolares si contemplamos la variable origen social (Barbería, 2009; Tarabini, Curran, Montes y Parcerisa, 2015).

El profesorado merece una consideración especial, porque como bien sabemos es un agente fundamental en el proceso de socialización del alumnado. Así, hemos de tener en cuenta: su origen y clase social; formación; antigüedad en el puesto; edad; género; años de permanencia en el centro; cercanía física y afectiva al centro; estabilidad laboral; puesto escolar (dirección, orientador/a, coordinador/a, tutor/a, ...); nivel educativo en el que imparte docencia y área de conocimiento; formación permanente; concepción del papel y las funciones de la institución educativa, las familias, grupo de iguales y medios de comunicación; percepción y justificaciones sobre las atribuciones de responsabilidades en la educación y las explicaciones sobre el rendimiento del alumnado: capacidad/esfuerzo, motivación, origen social, inmigrantes, familia, bar- rio/pueblo que van a incidir en cláusulas de salvaguarda, efecto Pigmalión, etnocentrismo (carencias culturales); opiniones sobre el poder/autoridad así como del compromiso docente; identificación con los principales problemas del centro. Además, no puede pasar desapercibido algunos aspectos vinculados a las percepciones que el profesorado tiene sobre la realidad escolar y como ello puede afectar a la experiencia escolar. En estudios anteriores (Cabrera, Cabrera, Pérez y Zamora, 2011) ya hemos constatado como este agente considera que los resultados académicos dependen mayoritariamente del alumnado (esfuerzo, motivación, implicación escolar...) y su familia (dedicación a sus hijos, implicación escolar...). Hasta el punto que los docentes consideran desde su experiencia profesional que si no cuentan con el apoyo de éstas y la motivación/el trabajo del alumnado ellos no pueden hacer nada. Asimismo, no se creen responsables ni de la escasa y en ocasiones nula implicación de éstas ni de la baja motivación/ expectativas del alumnado. Al mismo tiempo, se hallan totalmente desarmados ante el poder de los medios de comunicación, cuya influencia consideran totalmente negativa para poder desempeñar su labor, por los valores y modelos sociales que inculcan, al encontrarlos contrapuestos a los que la institución educativa transmite. También creen que en general es negativa la influencia del grupo de iguales pues los apartan "del correcto camino". Por tanto, se perciben "solos ante el peligro". Como nos señala Martín Criado (2004) y Resende (2010), todo lo que está fuera de la escuela es malo, y ellos tienen las manos atadas, pues si no cuentan con las condiciones óptimas para desempeñar su labor (políticas educativas realistas, centros escolares bien dotados, ratios adecuadas, familias y alumnado comprometido, "buenos" medios de comunicación) su labor es imposible. Sólo se salvan aquellos alumnos trabajadores, motivados y concienciados con las posibilidades que ofrece el sistema educativo.

Para delimitar la experiencia escolar del alumnado, diferenciamos una serie de facetas y dimensiones capaces de afrontar sus múltiples determinantes e influencias, contrastando las representaciones y justificaciones del alumnado en función del origen social, tipo de familia, género, lugar de procedencia, tipo de centro, y rendimiento educativo:

a) Los resultados educativos, es decir el fracaso, el éxito, el abandono.

b) Los procesos de exclusión, rechazo, resistencia, resignación, identificación expresiva e instrumental en relación con las percepciones.

c) Las trayectorias escolares y su opinión sobre sus capacidades y talentos.

d) Sus percepciones y justificaciones en relación a las opciones escolares. 
e) Sus motivaciones y expectativas a corto y medio alcance.

f) Su comportamiento y sus relaciones sociales en el aula y en el centro.

g) Su consideración sobre el currículum, los conocimientos y las tareas escolares (utilidad, interés).

h) Su valoración de los modelos docentes y los tipos ideales de alumnado.

i) El pensamiento acerca de la igualdad de oportunidades y la movilidad social a través de los estudios.

j) Sus valoraciones sobre las familias: clima afectivo, apoyo escolar y estilos educadores.

k) Las percepciones sobre "los iguales": procesos de identificación por género, tipo de amistades, conformación de grupos.

l) Sus opiniones sobre su lugar de residencia: barrios como lugares de estigmatización, zonas residenciales...

Los otros actores: De la escuela a la familia, los iguales y los medios de comunicación

En todo caso, nos interesa estudiar las experiencias escolares, no sólo para explicar los resultados académicos y posibles vías de intervención, sino también para establecer el papel que juegan en la conformación de las identidades juveniles.

El joven está siendo sometido a socializaciones múltiples y dispares que influyen en su experiencia escolar, como puede ser la familia, la escuela, los medios de comunicación el grupo de iguales y el barrio, que van conformando su personalidad e identidad juvenil y escolar.

La socialización familiar va a ser muy importante en la conformación de la identidad, tanto por su efectividad al ser la primera y estar vinculada al elemento afectivo como por su durabilidad a lo largo del tiempo. Esta socialización no va a ser homogénea, sino todo lo contrario, pues dependerá de distintos elementos que incluso varían su influencia a través del tiempo y según las diferentes situaciones a las que se encuentren sometidos. Así, puede variar según el sexo del agente socializado y socializador, la clase social, el tipo de familia (monoparental, nuclear, extensa, ensamblada o padres separados) y las personas que convivan en la casa (hermanos, abuelos, otros familiares), pues cada uno ellos actuará según sus diferentes experiencias sociales que estarán relacionadas con su género, la posición que ocupen en la casa y los modelos de atribución de roles; la situación laboral en la que se encuentren (estar desempleados o trabajando, estabilidad o inestabilidad laboral, precariedad...) y ocupación; así como según el nivel educativo de los progenitores, las relaciones sociales de los diferentes miembros de la familia, el tipo de ocio que realicen, etc.
De esta manera, a lo largo del proceso de investigación indagaremos sobre las siguientes dimensiones analíticas:

a) Las percepciones de las familias sobre sus prácticas educativas-socializadoras en relación a la trayectoria escolar de sus hijos/as: organización de tareas, clases particulares, asistencia al centro, etc.

b) Las expectativas y visiones de los progenitores sobre la institución escolar y sus exigencias: vislumbrar procesos de frustración, aceptación e indiferencia.

c) Las percepciones de las familias sobre la igualdad de oportunidades y la movilidad social a través de los estudios.

d) Las diferencias en información y conocimiento sobre "las reglas del mercado escolar" en función de las distintas configuraciones familiares y su clase social.

e) La probable relación entre el nivel de estudios de las familias, el relato sobre la institución educativa y las formas de configurar los procesos de socialización en los hogares.

f) Las visiones y expectativas de los padres y madres sobre el rendimiento, capacidades, motivación y comportamiento.

g) Los tipos de identificación con la escuela, los instrumentos y estrategias de reproducción y/o cambio. Posible relación de estas variables con las diferencias socioculturales de las familias.

La influencia de su grupo de iguales en el barrio, en el centro escolar y en las distintas agrupaciones/ asociaciones en las que participe (musicales, clubs de recreo, deportivas...). La influencia socializadora del entorno, aunque estrechamente, vinculada a la clase social del joven, puede variar dependiendo del tipo de barrio o zona en la que resida, de modo que sus características pueden producir una fuerte impronta social sobre la configuración de las identidades.

Los medios de comunicación juegan un papel importantísimo de cara a la conformación de su identidad: televisión, cine, música, internet donde el joven selecciona qué ver, escuchar, en dónde participar, cuándo y con quién.

\section{Conclusiones}

Nuestra perspectiva analítica no lleva a considerar que todo es relativo y que por tanto no puede haber ninguna política educativa que trabaje en pos de la igualdad de oportunidades educativas. Analizar en profundidad lo que está ocurriendo en el sistema educativo, buscar las regularidades, intersecciones y configuraciones que adoptan las experiencias escolares, profesionales y familiares atendiendo 
particularmente a la desigualdad económico-social, puede abrir vías para diseñar propuestas realistas. Al tiempo, y desde una óptica emancipadora, partimos de una visión holística de la realidad, en donde lo global no debe evitar estudiar, en relación con lo anterior, las realidades individuales y donde el estudio de esas realidades individuales no obvie la necesaria conexión y condicionamiento que esas realidades tienen de las estructuras sociales, económicas y políticas lo que hace imprescindible implementar políticas socio-económicas-culturales que contribuyan a la disminución de las desigualdades. Efectivamente sería ingenuo pensar que los problemas de las desigualdades sociales se solucionan exclusivamente a través de las políticas educativas. En cualquier caso, es necesaria una redefinición, desde una perspectiva comprometida con la justicia social, de nuevas lógicas y racionalidades para el sistema educativo, toda vez que se ha demostrado que la lógica de adaptación a las necesidades económicas es una cobertura ideológica que fortalece claramente la desigualad social.

\section{Notas}

1 Este análisis surge al amparo del marco teórico que estamos llevando a cabo en relación a una investigación denominada "Experiencias escolares y desigualdad social" dirigido por la profesora C. N. Pérez-Sánchez en la Universidad de La Laguna. Este estudio sociológico se centra, particularmente, en las experiencias escolares del alumnado de Educación Secundaria Obligatoria en el municipio de San Cristóbal de La Laguna en la isla de Tenerife (Islas Canarias)

2 Lo destacan 8 de cada 10 profesores encuestados. Cuestionarios propios pasados a unos 2.500 profesores y profesoras ( $10 \%$ del total).

3 La traducción española de esta obra se edita en 2010, aunque el original en francés previo a su obra en coautoría con Martucelli, es de 1994.

\section{Referencias bibliográficas}

Angus, L. (1993). The sociology of school effectiveness. Abingdon, UK: Carfax Publishing Co.

Ball, S. J. (1997). Good school/bad school: Paradox and fabrication. British Journal of Sociology of Education, 18(3), 317-336.

Barbería, J. L. (2009, 7 de abril). La clase perdedora. El País. Disponible en https://elpais.com/diario/2009/04/07/sociedad/1239055201_850215. html

Bernstein, B. (1988). Clases, códigos y control (Vol. I). Madrid: Akal.

Boltanski, L., y Chiapello, È. (2002). El nuevo espíritu del capitalismo. Madrid: Akal.

Bonal, X. (Dir.) (2005). Apropiaciones escolares: Usos y sentidos de la educación obligatoria en la adolescencia. Barcelona: Octaedro.
Bourdieu, P., y Passeron, C. (1977). La reproducción. Barcelona: Laia.

Brown, P., y Tannock, S. (2009). Education, meritocracy and the global war for talent. Journal of Education Policy, 24(4), 377-392.

Cabrera, B. (1991). Culturas sociales y culturas escolares en sociología de la educación. Revista Tempora, (17-18), pp. 25-56.

Cabrera, B., Cabrera, L., Pérez, C., y Zamora, B. (2011). La desigualdad legítima de la escuela justa. RASE, 4(1), 307-335.

Cabrera, B. (Dir.), Báez, B., Cabrera, L., Noda, M. M., Pérez, C. N., Santana, F., y Zamora, B. (2011). La compleja e inacabada constitución de grupos profesionales: Origen social, condiciones de vida, prácticas y mentalidades sociales y laborales del profesorado no universitario en Canarias (Informe técnico). Universidad de La Laguna, Instituto de Ciencias Políticas y Sociales. Disponible en https:// www.researchgate.net/publication/273336600_ La_compleja_e_inacabada_constitucion_de_grupos_profesionales_Origen_social_condiciones_de_ vida_practicas_y_mentalidades_sociales_y_laborales_del_profesorado_no_universitario_en_Canarias

Carmona Rodríguez, C. (2017). Tested: Una reflexión sobre medir en educación. RASE, 10(2), 268-282.

Collet Sabé, J. (2013). ¿Cómo y para qué educan las familias hoy? Los nuevos procesos de socialización familiar. Barcelona: Icaria.

Derouet, J.-L. (2002). A sociologia das desigualdades em educação posta à prova pela segunda explosão escolar: Deslocamento dos questionamentos e reinício da crítica. Revista Brasileira de Educação, (21), 5-16.

Derouet, J.-L. (2005). Repenser la justice en éducation. Education et Sociétés, 16(2), 29-40. doi: 10.3917/ es.016.0029

Derouet, J.-L., y Normand, R. (2011). Caesars and Rubicon: The hesitations of French policymakers in identifying a third way in education and training. Journal of Educational Administration and History, 43(2), 141-163.

Dubet, F. (2000). The sociology of pupils. Journal of Education Policy, 15(1), 93-104.

Dubet, F. (2010). Sociología de la experiencia. Madrid: Editorial Complutense-CIS.

Dubet, F., y Martuccelli, M. (1998). En la escuela. Sociología de la experiencia escolar. Buenos Aires: Losada.

Duschatzky, S. (1999). La escuela como frontera: Reflexiones sobre la experiencia escolar de jóvenes de sectores populares. Buenos Aires: Paidós Ibérica.

Fernández Enguita, M. (Ed.) (1997). Los desiguales resultados de las políticas igualitarias: Clase, género y etnia en la educación. En Sociología de las instituciones de educación secundaria (pp. 108-123). Barcelona: ICE.

Giró, J., y Andrés, S. (2016). Instalados en la queja: El profesorado ante la participación de las familias en la escuela. RASE, 9(3), 334-345. 
Grignon, C. (1991). La escuela y las culturas populares. Revista Archipiélago, (6), 15-19.

Kessler, G. (2002). La experiencia escolar fragmentada. Estudiantes y docentes en la escuela media en Buenos Aires. Buenos Aires: UNESCO.

Langa Rosado, D. (2006). Las experiencias académicas de los estudiantes universitarios y la clase social. Iniciación a la Investigación, (1), i-x.

Martín Criado, E. (2004). El idealismo como programa y como método de las reformas escolares. El Nudo de la Red, (3/4), 18-32.

Martín Criado, E., Gómez Bueno, C., Fernández Palomares, F., y Rodríguez Monge, A. (2000). Familias de clase obrera y escuela. Irún: Iralka.

Martín, R., y Bruquetas, C. (2014). La evolución de la importancia del capital escolar en la clase obrera. RASE, 7(2), 373-394.

Merino, R., García, M., Torrent, D., y Valls, O. (2017). Separación del alumnado por niveles en $4^{\circ}$ de ESO y complejidad social de los centros. Límites y posibilidades del curriculum comprensivo. Revista Tempora, (19), 181-198.

Núñez, P., y Litichever, L. (2015). Radiografías de la experiencia escolar. Ser joven (es) en la escuela. Buenos Aires: Grupo Editores Universitarios.
Pérez-Sánchez, C. N. (2002). Análisis sociológicos de las relaciones entre la cultura escolar y las culturas subalternas (Tesis de doctorado). Universidad de La Laguna, Santa Cruz de Tenerife, España.

Pérez-Sánchez, C. N., y Noda-Rodríguez, M. (2012) La familia en el punto de mira del profesorado. In X. Bonal, C. Díaz, y D. Luque (Orgs.), Actas de la XVI Conferencia de Sociología de la Educación (pp. 743-756). Madrid: Asociación de Sociología de la Educación.

Resende, J. M. (2010). A sociedade contra a escola?. Lisboa: Instituto Piaget.

Sambucceti, V. (2014). Estudio de las experiencias escolares discontinuas en los/las jóvenes de la clase trabajadora (Tesis de doctorado). Universidad Nacional de La Plata, Argentina.

Tarabini, A. (2015). La meritocracia en la mente del profesorado. RASE, 8(3), 349-360.

Tarabini, A., Curran, M., Montes, A., y Parcerisa, L. (2015). El rol de los centros educativos en la prevención del abandono escolar. Una aproximación desde la perspectiva micropolítica. Educação, Sociedade \& Culturas, (45), 121-141.

Willis, P. (1988). Aprendiendo a trabajar. Madrid: Akal.

Willis, P. (2008). Los soldados rasos de la modernidad. La dialéctica del consumo cultural y la escuela del siglo XXI. RASE, 1(3), 43-66.

Recebido a 03/10/2017. Aceite para publicação a 27/11/2017.

Francisco Santana-Armas (fsantana@ull.edu.es). Universidad de La Laguna, Departamento de Sociología y Antropología. Facultad de Educación, Campus Central, Módulo B, Apartado 456, 38200 San Cristóbal de La Laguna, $\mathrm{S} / \mathrm{C}$ de Tenerife, Islas Canarias.

María del Mar Noda-Rodríguez (mmnoda@ull.es). Universidad de La Laguna, Departamento de Sociología y Antropología. Facultad de Educación, Campus Central, Módulo B, Apartado 456, 38200 San Cristóbal de La Laguna, $\mathrm{S} / \mathrm{C}$ de Tenerife, Islas Canarias.

Carmen Nieves Pérez-Sánchez (cperez@ull.edu.es). Universidad de La Laguna, Departamento de Sociología y Antropología. Facultad de Educación, Campus Central, Módulo B, Apartado 456, 38200 San Cristóbal de La Laguna, $\mathrm{S} / \mathrm{C}$ de Tenerife, Islas Canarias. 